\title{
Bibliotheken als Orte der kulturellen Bildung
}

Fragen über das Verhältnis zwischen Bibliotheken und Kultur, d. h. über die Bibliothek als kulturellen Ort zu stellen, bedeutet das schließlich nicht, den Begriff »Bibliothek" selbst zu befragen? Wenn hinter dem Wort "Bildung " gleichzeitig die Kultur als eine Sammlung von Kenntnissen (Wissen) und die Vermittlung von Kenntnissen (das Wissen) als ein Grundbaustein der Erziehung darstellt, muss eines festgestellt werden: Die Bibliothek ist der Inbegriff der kulturellen Bildung, da das Buch (zumindest bis heute) das Hauptmedium ist, das den Erwerb der Kultur ermöglicht - und dank dieses Erwerbs kann sich dann jeder buchstäblich intellektuell ausbilden. Die Bibliothek ist also wesensmäßig ein Ort der Kultur, da sie Sammlungen von Büchern und Drucksachen jeglicher Art bewahrt. Die Bibliothek ist aber zunächst ein Ort, der als Ort seine Funktion verrät und selbst zum kulturellen Objekt wird, so zum Beispiel der berühmte Raum, der im Vatikanpalast Papst Julius II. als Bibliothek und Arbeitszimmer diente. Jeder kennt diese "Stanza della Signatura» mit den Fresken von Raffael ausgestattet (darunter die "Schule von Athen»), wo sich die Ordnung der Welt und die Ordnung des Wissens verbinden und das Chaos überwinden. Was die Bücher möglich machen, das zeigt das Fresko, und dieses Verfahren zeichnet den Ort der Bibliothek als einen Ort der Kultur aus, der als solcher bestehen bleibt, auch wenn er seine ursprüngliche Funktion verloren hat.

Wir haben hier ein schönes Beispiel einer Bibliothek als kulturellen Ort - einer Bibliothek, die zum Museum geworden ist. Und trotzdem war die Bibliothek für die meisten Menschen lange Zeit eher ein Ort, der mit der Wissenschaft und der intellektuellen Arbeit verbunden war, wenn ein »kultureller Ort« im strengen Sinne eher mit Muse und Entspannung verbunden ist - denken wir an Kinos, Theater oder Museen. Die Bibliothek ist ursprünglich ein kultureller Ort der anderen Art. Wenn das Bibliotheksgebäude, ab der Mitte des 19. und während des ganzen 20. Jahrhunderts, zu einem selbstständigen Bau wird, wird dieser eher als ein Wissensspeicher wahrgenommen (dessen

1) Vera Trost, "L'»art dans I'architecture " à la Bibliothèque régionale du Wurtemberg: I'œuvre graphique de Josua Reichert", in: La Revue de la BNU, Nr 1, 2010, S. 34-43.
Ausstattung überhaupt funktionsgerecht gedacht wird). Es ist ein Ort der Wissenschaft, viel mehr als ein Ort der Kultur im Sinne von »kulturellen Aktivitäten « - letztere werden in den oben erwähnten »kulturellen Orten « betrieben. Und trotzdem ist die kulturelle Bestimmung der Bibliotheken unleugbar - und das seit Jahrhunderten. Es sind Orte, wo die Kultur sich zeigt, und das, vor allem, in den Gebäuden selbst: Die Bibliothek kann in sich ein Kunstwerk sein. Bleiben wir in Schwaben: Wer fährt nach Bad Schussenried oder nach Ulm-Wiblingen, um dort ein Buch zu lesen? Aber auch in Orten, die man aufsucht, um Bücher zu lesen, kann eine kulturelle Dimension (im Sinne einer von künstlerischer Ergriffenheit hervorgerufenen Bereicherung) dort selbst erlebt werden. Im Rahmen dieses Aufsatzes ist es nicht die Stelle, es ausführlich darzulegen, aber ich möchte trotzdem ein Beispiel aus Stuttgart nehmen - nämlich den Dialog, der zwischen den Bibliothekaren, der Landesverwaltung und den Künstlern HAP Grieshaber und Josua Reichert anlässlich des Neubaus der Württembergischen Landesbibliothek nach dem Zweiten Weltkrieg stattgefunden hat. Dieser Dialog hatte eine musterhafte »Kunst am Bau « zur Folge, die so versinnbildlicht geworden ist, dass man schreiben konnte: "Josua Reicherts "Stuttgarter Drucke" sind untrennbar mit der Württembergischen Landesbibliothek verbunden «". Das Reichertsche Programm beruht auf der Vielfalt der Schriften, der Sprachen und der literarischen Quellen; es ist in sich eine Art symbolischer "Bildung ", sodass es mir nicht ganz ungeeignet scheint, das, was in Stuttgart in den 60er Jahren in Gang war, mit dem zu vergleichen, was in Rom am Anfang des 16. Jahrhunderts zwischen Julius II., seinen Räten und Raffael betrieben wurde - natürlich in einer modernen, demokratischen und auch bescheidenen Weise...

So veredelnd es scheinen mag, kann aber die kulturelle Tätigkeit einer Bibliothek, ihr Image als kultureller Ort, sich nicht auf die Hervorhebung eines prestigeträchtigen Baus beschränken. Für eine Bibliothek, die ein Kulturerbe zu pflegen hat (von solchen ist hier die Rede), ist die Bewahrung 
von Dokumenten »ein edles Ziel nur, wenn letztere in den Vordergrund gestellt werden, zur Geltung gebracht werden «². "Die Bibliothek, der Archivspeicher, all diese Einrichtungen, die Dokumente sammeln, müssen auch deren Inhalte zurückgeben, in der einfachen Form der Ausleihe oder (was natürlich komplizierter ist) in der Inszenierung ihres Wissens - ein Wissen, das manchmal verkannt ist ${ }^{3}$. Hier befindet sich, meiner Meinung nach, das Herz der kulturellen Tätigkeit der Bibliotheken - überhaupt in einer Zeit wie der unsrigen, die eine Zeit der Bilder ist, wo selbst die Wissenschaft Bilder braucht, zum Gegenstand der Ausstellung zu werden wünscht.

Nun habe ich es endlich gesagt, das Stichwort "Ausstellung". Im 16. Jahrhundert inszeniert sich die Wissenschaft in Fresken, aber sie sehnt sich heute immer noch nach Inszenierungen, natürlich in einer verschiedenen Form, die man unter dem heute so sehr in Mode geratenen Begriff der "Mediation « (oder Vermittlung) zusammenfassen kann. Diese Inszenierung nimmt in den meisten Fällen die Form der Ausstellung an - und an Ausstellungen denkt des Öfteren das breite Publikum, wenn von der kulturellen Tätigkeit der Bibliotheken die Rede ist. Wir hatten diesem Thema eine Nummer der Revue de la BNU gewidmet ${ }^{4}$, mit Beiträgen aus dem eigenen Haus sowie mit anderen Annäherungen über diese "kulturelle Mediation « (oder Kulturvermittlung) - anders gesagt, wie man dank einer Ausstellung ein Werk, einen Gedanken klarer erscheinen lässt und damit besser verständlich macht. Der Begriff Bibliotheksausstellung ist im Laufe des 20. Jahrhunderts groß geworden (wir dürfen nicht vergessen, dass man in Frankreich die Bibliotheken erst ab den 70er-80er Jahren als mögliche museale Orte - und das nur vereinzelt betrachtet hat, wie D.-G. Picard in der Revue de la BNU, Nr. 3, 2011, betont ${ }^{5}$. Er ist heutzutage zum Inbegriff ihrer kulturellen Tätigkeit geworden. Ein retrospektiver Blick über den Fall Bibliothèque nationale et universitaire de Strasbourg (BNU) zeigt, dass sie eher ein Vorreiter war: "während 80 Jahren [das heißt von den 30er Jahren bis 2011] hat die BNU im Rahmen ihrer kulturellen Politik mehr als 100 Ausstellungen organisiert « ${ }^{6}$. Diese Feststellung zeigt, dass die BNU auf eine wirklich lange Tradition zurückblicken kann; eine Beschleunigung in dieser Politik der "Mediation in der Bibliothek « am Ende des 20. und am Anfang des 21. Jahrhunderts

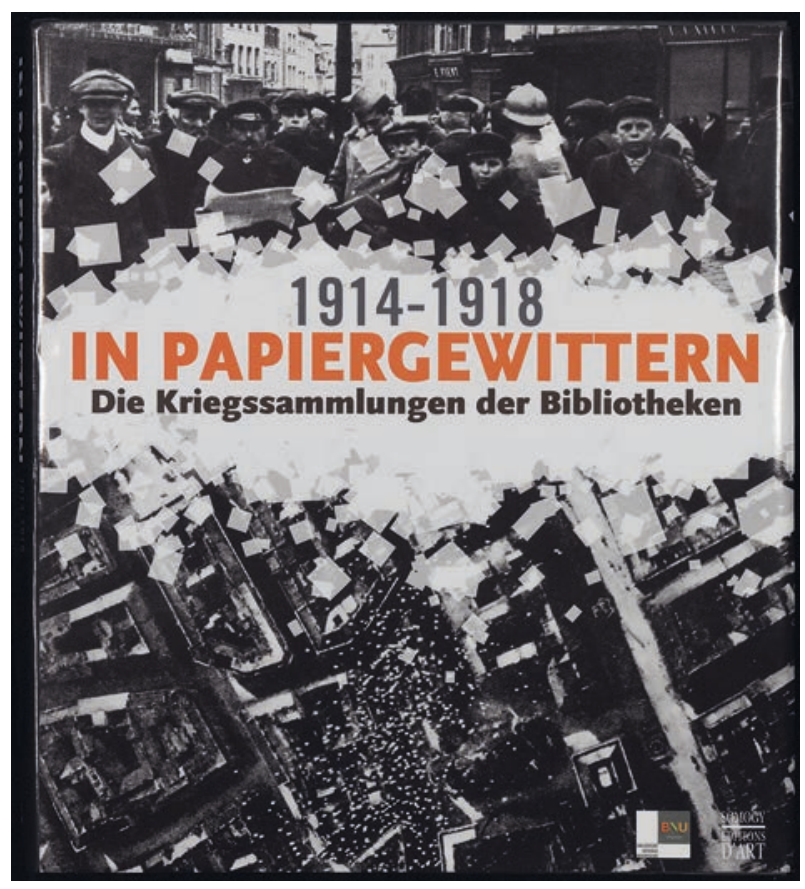

Abb. 1: Die Ausstellung "Orages de papier" (2008), ein Kooperationsprojekt zwischen BNU, BnF und WLB, wurde auch 2009 in Stuttgart unter dem Titel "In Papiergewittern" gezeigt. (C) Emmanuel Somot

muss trotzdem unterstrichen werden. Es schien von nun an nicht mehr möglich, sich auf eine traditionelle Mediation zu beschränken - eine Mediation, die sich auf einen pädagogischen Gebrauch eines ausgeliehenen und gelesenen Buches stützt.

Aber zurück zur Württembergischen Landesbibliothek, deren jüngste Geschichte uns einen weiteren Schritt auf dem Weg zur "Mediation « zeigt. "Das in der Bibliothek gesammelte "Gedächtnis der Menschheit" sollte nicht nur in den verschiedenen Lesesälen und Magazinen bereitstehen, sondern auch in einem „Buchmuseum" vermittelt werden " (Wilhelm Hoffmann, Direktor der WLB in den 60er Jahren 7 ). Diese museale Dimension der Bibliotheken, die ein Kulturerbe betreuen, ist ein Wahrzeichen unserer Zeit (auch wenn die Beispiele der BNU und der WLB zeigen, dass es einige Vorläufer gab). Das ist in Frankreich sehr auffallend: ich möchte an dieser Stelle nur das anspruchsvolle Projekt der Bibliothèque nationale de France erwähnen, nämlich »Richelieu: Bibliothèques, Musée, Galeries", an ihrem historischen Standort im

2) Christophe Didier, Albert Poirot, "Éditorial", in: La Revue de la BNU, Nr 3, 2011, S. 5.

3) Ibidem

4) Nr 3, "Exposer" (2011), bald online unter https://journals.openedition. org/rbnul

5) David-Georges Picard, "Cent expositions à la BNU : un début d'historiographie», in: La Revue de la BNU, Nr 3, 2011, S. 8-17.

6) D.-G. Picard, op. cit.

7) Zitiert von Vera Trost, in art. cit. 
Zentrum von Paris geplant... oder sogar das Renovationsprogramm der BNU, das zwischen 2010 und 2014 stattfand. Dort wurden neben einem großen Ausstellungsraum museale Räume vorgesehen, die für die Öffentlichkeit geöffnet werden sollten: die dort aufbewahrten Dokumente stehen einem neugierigen und bewundernden breiten Publikum zur Verfügung - ihre ursprüngliche Funktion als »Informationsträger « behalten sie aber natürlich, da sie für Forscher immer zugänglich bleiben.

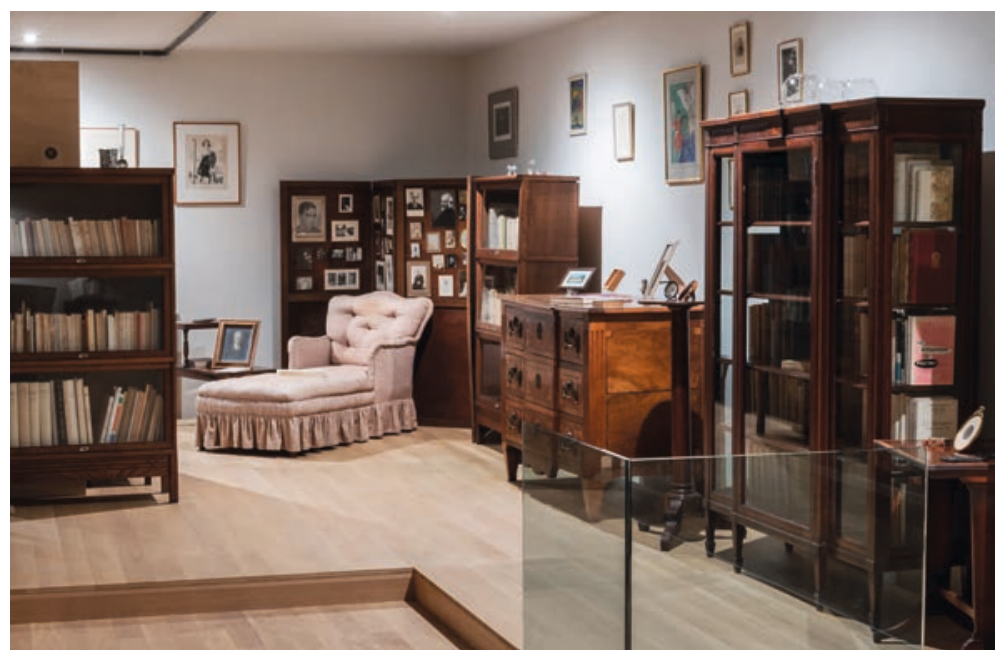

Abb. 2: Ein Blick auf die musealen Teile („réserves visitables") der BNU. (C) BNU-JPR

Soweit für Frankreich - aber ähnliches gilt für Deutschland: es sollen hier als einziges Beispiel die jüngsten musealen Einrichtungen der Deutschen Bücherei in Leipzig erwähnt werden.

Es handelt sich, meiner Meinung nach, um eine gesellschaftliche Entwicklung: die Wissenschaft (es sei hier sowohl Mathematik, Physik oder Medizin als auch Geisteswissenschaften gemeint) will sich zeigen, sich verständlich machen. Das Museum als eine beim breiteren Publikum beliebte Form der »kulturellen Mediation « scheint am besten geeignet, um dieses Ziel zu erreichen: heute sieht man sogar "Universitätsmuseen ", wie z. B. den 2008 in Strasbourg gegründeten "Jardin des sciences" (Garten der Wissenschaften), dessen erklärte Absicht es

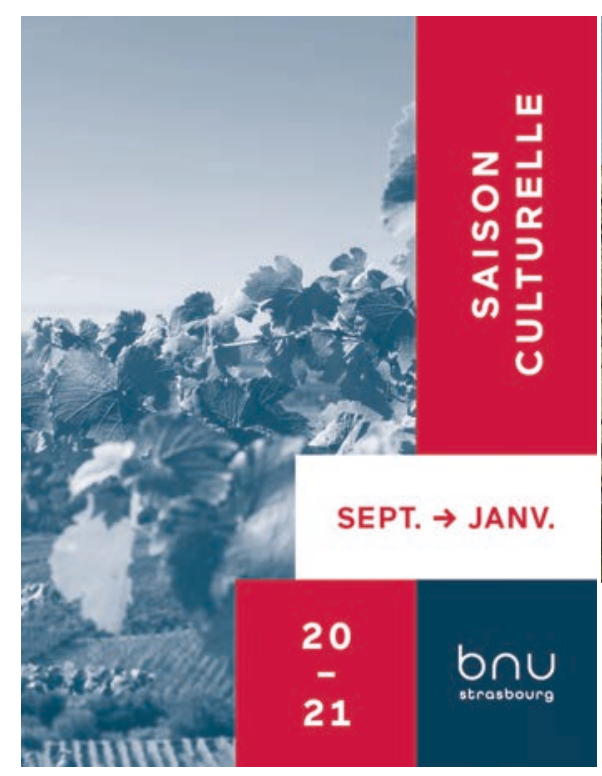

Zoologie, von der Paläontologie bis in die Ägyptologie reichen, besser zur Geltung zu bringen. Das endgültige Ziel? "Die Wissenschaft durch ihr aufbewahrtes Erbe besser sichtbar zu machen « ${ }^{8}$. Gilt ähnliches nicht für Bibliotheken, die ihr aufbewahrtes Wissen in der Form eines Kulturerbes ausstellen, wenn sie die hinterlassenen Spuren zeigen, an denen dieses Wissen unmittelbar greifbar ist? Über Mediation wurde oft geredet - aber könnte es anders sein? Dieser Begriff ist jetzt mehr als eine Mode, er ist zum Motto westlicher Gesellschaften geworden, die scheinbar am meisten die soziale Ausgrenzung fürchten. Für diese ist eine "Kultur der Inklusion « eine der unbedingten Voraussetzungen des sozialen Zusammenhalts. Mit der zunehmenden Studentenzahl sind Bibliotheken beliebte Orte des Geselligkeit geworden; sie befinden sich also oft in erster Reihe (zusammen mit Museen, Theatern oder Opernhäusern) und werden oft von den staatlichen (oder städtischen) Amtsträgern ermuntert, als "Orte der Mediation " zu funktionieren. Sie sind nicht nur Orte, wo man sich mit anderen um des Wissens willen trifft (z. B. in einem Lesesaal), sie werden auch zu Orten der Geselligkeit rund um eine Veranstaltung - wenn nicht ein "Event" -, in einem Vortragsraum, einem Foyer, anlässlich einer öffentlichen Führung oder eines Tages der offenen Türen... Es genügt nicht zu zeigen, was man hat, man muss hin locken, dann begleiten. Dies hat auch zur Folge, so scheint es mir, dass von nun an auch in Bibliotheken sogenannte "kulturelle Saisons" entstanden sind.

ist, Sammlungen, die von der Mineralogie bis in die 
Die BNU scheint mir an dieser Stelle beispielhaft, was diese Entwicklung der Bibliotheken als »Orte der Kultur « angeht. Sie betrieb zwar, wie gesagt, seit langer Zeit eine richtige kulturelle Politik, die verschiedene Formen annahm (nicht nur Ausstellungen, sondern auch Vorträge, Vorlesungen, kleine Konzerte oder Tage der offenen Türen); diese Politik wurde aber erst am Anfang des 21. Jahrhunderts im Organigramm der Bibliothek sichtbar, im Jahr 2007 mit der Schaffung eines spezifischen Teams (des "Service de l'action culturelle»), das heute sieben Personen zählt. Anspruchsvolle Ausstellungen, mit eigenen Szenographien und gedruckten Ausstellungskatalogen, haben im Laufe der Zeit (vor allem seit 2003 und der Ausstellung Impressions d'Europe - die auch in der WLB gezeigt wurde!) die Entwicklung von Begleitprogrammen ermöglicht, die Führungen (manchmal in Fremdsprachen), Vorträge, Theaterstücke, Konzerte usw. anbieten. Informationen über solche Veranstaltungen gab es am Anfang nur auf Flyern oder Extra-Programmen; seit 2016 aber veröffentlicht die BNU zweimal im Jahr, im September und im Januar, ein Generalprogramm (gedruckt und online $\left.^{9}\right)$ aller vorgesehenen Veranstaltungen. Dieses Heftchen ist seit vier Jahren immer (ein bisschen) dicker geworden, da in der Direktion der BNU der oben schon erwähnte Wille besteht, die Bibliothek mehr nach außen zu öffnen, weit über die »klassische» Leserschaft (Studenten, Akademiker, Forscher usw.) - und auch weit über die Ausstellung als zusätzliche Attraktion hinaus. Die BNU ist also jetzt ein fester Partner nicht nur der Straßburger Universität, sondern auch eines städtischen Kinos (dessen Bibliothek in ihre Bestände einverleibt wurde), eines Festivals für Musik des 20. Jahrhunderts, der städtischen Oper, der städtischen Museen sowie von verschiedenen künstlerischen und kulturellen Verbänden. Das Ergebnis: eine vielfältige "Saison «, wo das Buch und das gedruckte Material nicht immer die Hauptrolle spielen.

Man sollte aber nicht denken, dass ich mich mit Selbstzufriedenheit darüber äußere. Die BNU ist inmitten der Innenstadt gelegen, wie in Stuttgart auf einer Kulturmeile, wo sich Bibliothek, Theater und Opernhaus in unmittelbarer Nähe befinden; sie ist auch seit 2014 sieben Tage in der Woche geöffnet. Dass eine solche öffentliche Einrichtung eine reiche Kultursaison anbieten kann, erscheint zweifellos selbstverständlich, und einen Weg zurück würde freilich niemand verstehen. Ein Risiko besteht trotzdem, meiner Meinung nach, wenn die kulturelle Politik sozusagen durchgeht und außer Kontrolle gerät: dass sie nicht mehr mit dem verknüpft wird, was Herz, Kernstück und Daseinsberechtigung einer Bibliothek bleibt - damit meine ich natürlich ihre Sammlungen.

Es geht hier um Gleichgewicht; für eine große Bibliothek, die ein Kulturerbe betreut, ist es nicht so schwierig, ihre kulturelle Politik auf das Hervorheben ihrer Sammlungen zu basieren - aber für welches Publikum? Das Schrifttum auszustellen ist immer eine Herausforderung, das weiß jeder Bibliothekar. Es ist auch sehr einfach, die zahlreichen Vorschläge, die man von der kulturellen Szene regelmäßig bekommt, mit ja zu beantworten und Veranstaltungen zu organisieren (Filme, Vorträge über das Tagesgeschehen, Konzerte usw.) die zwar ein neues Publikum in die Bibliothek anlocken werden - aber wo steckt der Vorteil, was das Hervorheben der Sammlungen angeht? Anders gesagt, ist es ein Vorteil für eine Bibliothek, wenn sie für einen Abend ein Publikum lockt, das aber die Bibliothek vielleicht kein zweites Mal benutzen wird? Der Weg, der zum Aufblühen der kulturellen Tätigkeit in den Bibliotheken führt, ist also ein schmaler Grat. In den verschiedenen "call for projects « die die EU (insbesondere im Rahmen des H2020Programms) finanziert, steht seit einigen Jahren eine wichtige Bedingung: dass man sich (auf gut europäischem Globish...) um die "Dissemination " kümmern muss. Was bedeutet das? Das Wissen, das Bibliotheken (und Bibliothekare) häufen, muss sozusagen alle Schichten der Gesellschaft durchfluten und sich nicht in den Elfenbeintürmen der Gelehrten stapeln - was man in der Universität Straßburg »den Dialog zwischen Universität und Öffentlichkeit neu denken« nennt. So organisiert die BNU z. B. seit 2016 Veranstaltungen mit dem Titel "Savoirs en partage«, wo akademische Forscher einem breiteren Publikum die Ergebnisse ihrer Arbeit vorstellen und darüber entspannt diskutieren. Solche Initiativen findet man natürlich auch anderswo: "Savoirs en partage" auf Deutsch, heißt es nicht... Wissen teilen ${ }^{10}$ 


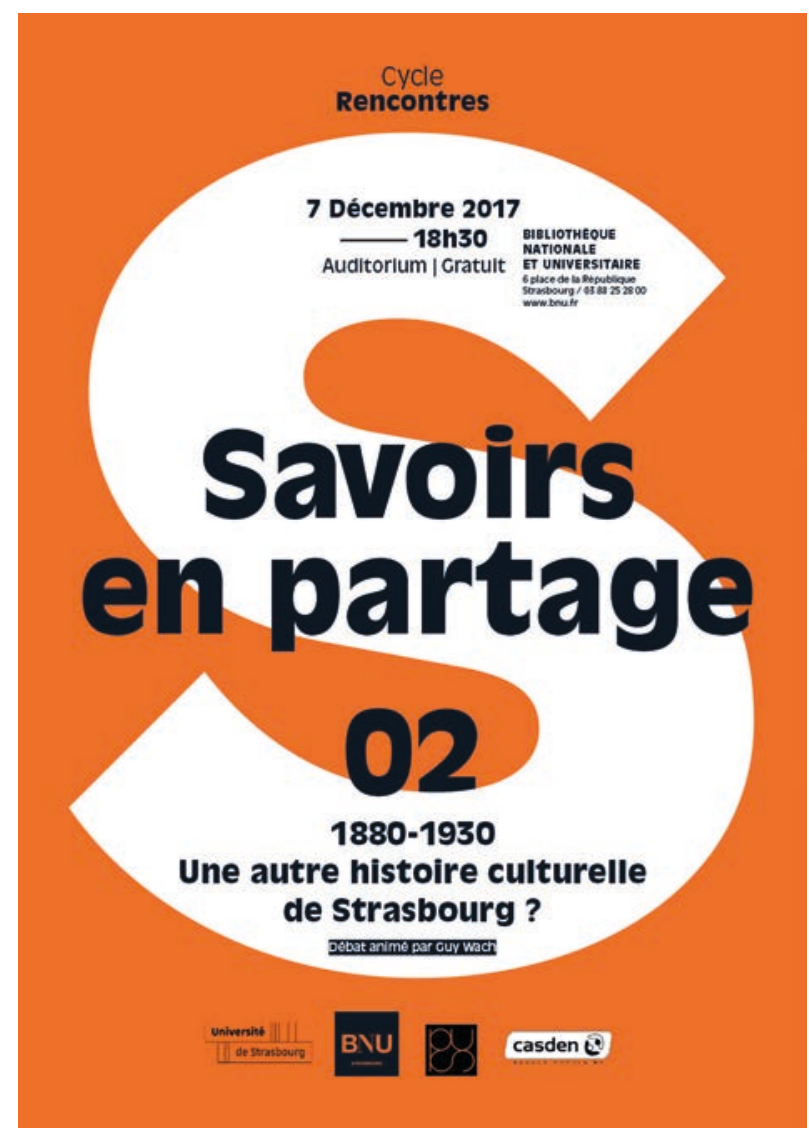

Abb. 5: Plakat der Veranstaltung "Savoirs en partage". Im Rahmen dieses Zyklus finden jährlich 3 bis 5 Veranstaltungen statt. (C) Brokism - Hugues François vollem Recht, es gibt keinen Vergleich zwischen der Betrachtung der "realen " Mona Lisa und dem besten Digitalisat von Da Vincis Meisterwerk; geht es anders mit Gutenbergs Bibel oder einer Handschrift von Hölderlin?

Die Mediation, die eine kulturelle Tätigkeit ermöglicht, kann sicherlich zur sozialen Inklusion beitragen, und für Bibliotheken und Bücher ein neues Publikum gewinnen - ein Publikum, das sonst vielleicht nie gekommen wäre. Diese Mediation scheint mir aber noch hoffnungsvoller, wenn das Ergebnis das Entstehen einer Gemeinschaft der Benutzer (»users community«) zur Folge hat einer Gemeinschaft, die die Kultur für wichtig hält und die immer noch in Bibliotheken geht, auch wenn die Zeit des Studiums vorbei ist, auch wenn die Bibliothek kein unmittelbares Bedürfnis mehr bedeutet. In diesem Sinne, so scheint es mir, sind Bibliotheken wirkliche "Orte der kulturellen Bildung ". So trägt die Bibliothek am Besten zur Bildung bei, in der die Kultur die Grundlage der Erziehung ist.

Christophe Didier
Eines möchte ich zum Schluss sagen: die Bibliothek darf kein bloßer "Ort, wo Veranstaltungen stattfinden « werden, das scheint mir entscheidend. Wenn man die "Erwerbungsabteilung" (die für die Arbeit der Katalogisierung, der Sacherschließung und der Ausleihe zuständig ist) der Stabsstelle „Kulturelle Politik (Synonym für Muße und pure Entspannung) gegenüberstellt, dann führt es zu einer Sackgasse. Eine weitere Sackgasse: die virtuelle Bibliothek (für den Forscher von seinem Arbeitszimmer mit allen nötigen Ressourcen sofort erreichbar) als "Exzellenzbibliothek« dem realen Ort gegenüberzustellen - einem realen Ort, wo nur Studenten (und einige Außenseiter...) sich befänden, die eine komfortable und kostenlose Zuflucht genießen, sich aber für die Sammlungen nicht interessieren. Das wäre das Ende der Bibliothek als ein Ort des Wissens, wo man das Wissen teilt. Bibliotheken müssen ihre Sammlungen lebendig halten, im Internet natürlich dank der Möglichkeiten der digitalen Sammlungen, immer und überall auf der Erde erreichbar, aber auch intra muros, wo man sie erklären, inszenieren und verständlich machen kann. Es wurde oft gesagt, und das, glaube ich, mit 